\title{
Amyloidosis cutis dyschromica
}

\author{
Jianjun Qiao ${ }^{1}$, Hong Fang ${ }^{1 *}$ and Hongtian Yao ${ }^{2}$
}

\begin{abstract}
Background: Amyloidosis cutis dyschromica is a rarely documented variant of cutaneous amyloidosis. To date, only 26 cases have been reported.

Objective: The purpose of this study was to improve the clinical and histopathological data for this variant of amyloidosis and to highlight the immunohistochemical features of the disease. The published cases were also reviewed.
\end{abstract}

Methods: We performed a retrospective review of patients with amyloidosis cutis dyschromica in a single centre. The clinical, histopathological and immunohistochemical features were documented and analysed.

Observations: We described 10 cases of amyloidosis cutis dyschromica. Six of them were female. Five patients were from the same family, and the other 5 were sporadic. The distinguishing features of the clinical presentation included generalised mottled hyper- and hypopigmented macules, which were asymptomatic or mild pruritic. The typical onset of the lesions occurred in childhood $(n=7)$ and occasionally after puberty $(n=3)$. No evidence of systemic amyloidosis deposition was observed in these cases of amyloidosis cutis dyschromica. Amyloid deposits were observed in the papillary dermis and were positive for the Congo red stain. An immunohistochemical study showed that the amyloid expresses cytokeratins CK34ßE12 and CK5/6.

Conclusions: We described the largest series of amyloidosis cutis dyschromica to date and reviewed the published patients. This rare disease is featured by generalised mottled hyper-and hypopigmented lesions, and it is a rare variant of primary cutaneous amyloidosis without evidence of systemic amyloid deposition. Positive staining for the cytokeratins CK34ßE12 and CK5/6 in amyloidosis cutis dyschromica suggests that the amyloid is derived from keratinocytes.

Keywords: Amyloidosis cutis dyschromica, Amyloid, Pigmentation disorder, Cytokeratin, Congo red, Hereditary disease

\section{Background}

Primary cutaneous amyloidosis refers to the extracellular deposition of amyloid material in previously apparent normal skin without systemic involvement. The major variants of primary cutaneous amyloidosis are macular and lichen [1]. Amyloidosis cutis dyschromica (ACD), first described in 1970, is a rarely documented variant of cutaneous amyloidosis [2]. Morishima described its features as the following: (i) dotted, reticular hyperpigmentation with hypopigmented macules distributed over nearly all of the body, (ii) no or little itch, (iii) onset before puberty and (iv) focal amyloid deposition under the

\footnotetext{
* Correspondence: fanghongzy@sina.com

'Department of Dermatology, The First Affiliated Hospital, College of Medicine, Zhejiang University, No. 79, Qingchun Road, Hangzhou 310003,

Zhejiang Province, China

Full list of author information is available at the end of the article
}

epidermis [2]. To date, there are only 26 published cases worldwide. Some recently published ACD cases presented with new characteristics. However, the origin of the amyloid was not described in most of these published cases.

To further understand this disease, we report 10 new cases of ACD and investigate the origin of the amyloid materials. We also review the cases of ACD reported in the literature.

\section{Patients and methods}

We performed a retrospective case analysis of 10 patients with ACD who were referred to our dermatology department from 2004 to 2012. We retrieved the patients' clinical histories, clinical findings, laboratory results and attending physician names. All skin biopsy samples were

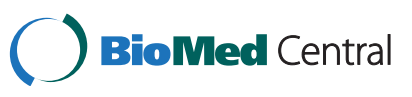


fixed in $10 \%$ formalin and embedded in paraffin. Four$\mu \mathrm{m}$-thick sections were stained with hematoxylin-eosin and Congo red. A panel of cytokeratin stains (Additional file 1: Table S1), including AE1/AE3, CK5/6 and CK34.BE12, was performed on the available tissues. PubMed and Google Scholar were searched for published cases in English. The study was approved by the Ethical Committee of The First Affiliated Hospital, College of Medicine, Zhejiang University. All patients gave their written informed consent.

\section{Results}

\section{Demographic features}

A total of 10 patients with ACD were identified in our database search from 2004 to 2012 (Additional file 2: Table S2). Four patients were male, and 6 were female. The patient age ranged from 17 to 73 years, with an average age of 38.7 years.

\section{Clinical presentation}

The clinical features of the 10 patients are presented in Additional file 2: Table S2. Patients no. 1a to no. 1e were from the same family. There were no family members with similar disease for the other 5 patients. All patients were born to non-consanguineous parents. They had no photosensitivity, blistering eruption or short stature and reported no history of extensive sun exposure, any other cutaneous disorder or systemic disease before the onset of the lesions.

The cutaneous lesions were asymptomatic or mild pruritic. The typical lesion onset occurred in childhood $(n=7)$ and occasionally after puberty $(n=3)$. The eruptions progressed gradually over years to involve almost the entire body and stabilised thereafter. The time elapsed from the initial presentation to the final diagnosis varied from 4 years to more than 50 years. The distinguishing features of the clinical presentation included generalised mottled hyper- and hypopigmented macules (Figure 1A, 1B and 1C). Diffuse hyperpigmented small papules were noted in one patient (Figure 2A, 2B). The size of the macules and papules ranged from 2 to $10 \mathrm{~mm}$, and they involved almost the entire body in a symmetrical pattern. The lesions were more pronounced on the trunk and the limbs with relative sparing of the face, hands, feet and neck in all cases (Figure 1C). There was no telangiectasia, erythema, or atrophy. The nails, hair, teeth, mucous membranes, soles and palms had a normal appearance. The systematic examination was unremarkable except for one patient with colon cancer.

Laboratory investigations, including blood cell count, urine analysis, abdominal ultrasonography, and serum protein electrophoresis, revealed no abnormality.

\section{Histopathological findings}

The histopathological findings of specimens from hyperand hypopigmented lesions in all cases indicated homogenous eosinophilic masses in the papillary dermis and sparse melanophages in the superficial dermis (Figure 3A). In addition to the features observed in the hyper- and hypopigmented lesions, specimens from the lichenoid papules showed mild hyperkeratosis. There were no significant alterations in the reticular dermis. The eosinophilic material stained positive for Congo red in all cases (Figure 3B). Similar cytokeratin staining profiles were found in all cases, with strong positive staining for CK34 $\beta E 12$ and CK5/6 and faint positivity or absence of staining for AE1/AE3 (Figure 4A, B).

\section{Treatment and outcome}

Oral vitamin $\mathrm{E}$ and vitamin $\mathrm{C}$ were the treatment of choice in most cases and resulted in minimal improvement. Three patients received oral acitretin at $20 \mathrm{mg}$ per day; two had a good response within three months, but the other demonstrated minimal improvement.

\section{Literature findings}

The literature data are summarised in Additional file 3: Table S3. A total of 26 published cases were identified in

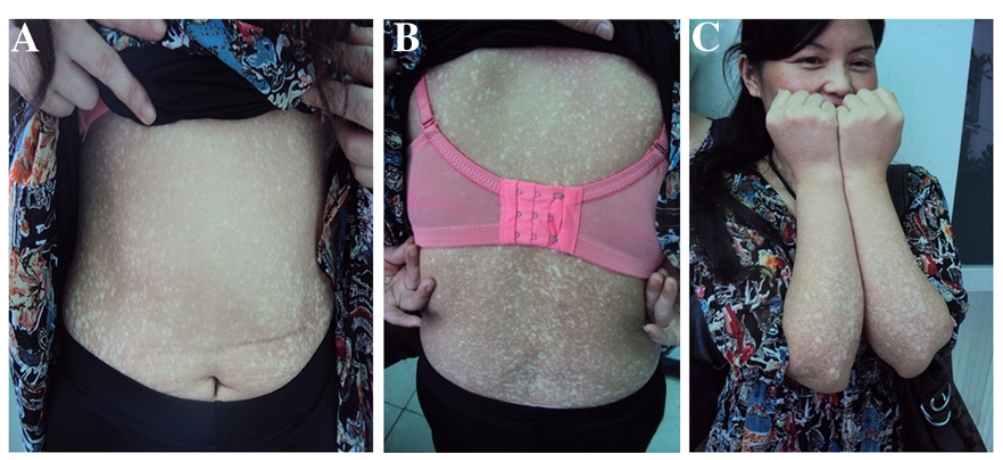

Figure 1 Clinical presentation of amyloidosis cutis dyschromica. A and B, Generalised mottled hyper- and hypopigmented macules were distributed over the entire body (patient 1b). C, However, the lesions on the face and distal extremities were mild. 

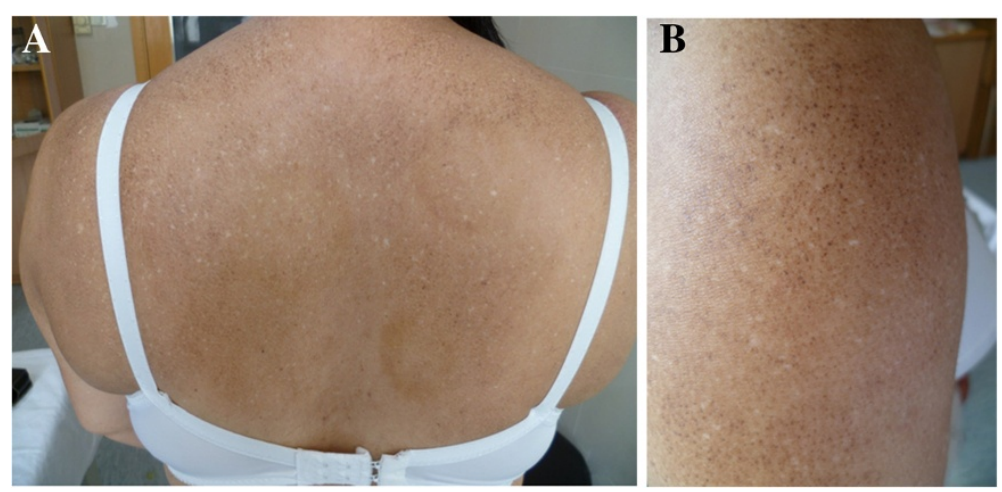

Figure 2 Clinical presentation of amyloidosis cutis dyschromica. In addition to the hyper- and hypopigmented macules, diffuse hyperpigmented small papules were noted (patient 4).

a literature search [2-17]. The ethnic distribution of the 26 cases is shown in Figure 5. Four patients did not have family members with the disease, and the other 21 cases were familial and distributed among 12 families.

\section{Discussion}

We report the largest series of ACD cases to date. Similar to macular amyloidosis and lichen amyloidosis, the Asian population is more susceptible to this disease, particularly Chinese, Japanese and Thai individuals. ACD was familial in 19 of 36 patients (10 in this series and 26 in the literature); 17 cases were sporadic. However, only two siblings had consanguineous parents. The onset of dyschromia occurred before puberty in most patients and after puberty in only 5 patients ( 3 patients in our series and 2 patients in the literature).

Itch is a significant symptom in patients with macular amyloidosis and lichen amyloidosis, which are the two most common variants of primary cutaneous amyloidosis [18]. The lesions of ACD are generally asymptomatic, but patients may express cosmetic concerns. Only 5 patients had a mild itch ( 2 in our series, 3 in the literature).

In addition to the characteristic generalised reticular hyperpigmentation checked with hypopigmented macules, small hyperpigmented papules were found in 5 patients ( 1 in our series, 5 in the literature). A clinical morphology overlap between ACD and other variants of primary cutaneous amyloidosis has been observed in published cases $[3,6,13]$. Presentations of blister $(n=2)$ [3], freckles $(n=1)$ [13] and telangiectasia $(n=2)$ [13] have been described. Concurrent with ACD with lichen, poikiloderma-like, dyschromic and bullous variant of primary cutaneous amyloidosis was reported in one patient [6]. Our series lacked any concurrent mucocutaneous disorders, and the hair, nails, teeth, membranes, palms, and soles were normal in all of our patients. Erythema was not found in any of the ACD patients.
Although ACD is widely accepted as a primary cutaneous amyloidosis, it has been associated with other diseases in a few cases. One case of ACD with generalised morphea was reported [13]. Two siblings presented ACD associated with atypical Parkinsonism, spasticity and motor weakness [5]. Systemic amyloid deposition could potentially explain the extracutaneous manifestations of the siblings, but neurological investigations revealed no evidence of systemic amyloid deposition [5]. The authors postulate that the syndrome in this family may have been caused by a concomitant mutation of closely linked genes [5]. In our series, colon carcinoma was found in one patient when he was 16 years old. The mechanism of the association is unknown. An ACD diagnosis may cause concern for treating clinicians because of the possibility of underlying systemic amyloidosis. However, no systemic involvement was detected in any of the reported ACD patients.

The histopathological findings in our series are similar to those reported in the literature, with amyloid depositing in the papillary dermis. Amyloid was observed in the superficial dermis in 2 published cases [10,18]. It is not clear why the amyloid deposition was so extensive in the two patients. Histopathologically papular ACD lesions also revealed amyloid deposits in the papillary dermis.

The presentation of melanophages in the superficial dermis suggests damage to the basal epidermis in ACD, lichen amyloidosis and macular amyloidosis. Although reports examining the origin of ACD amyloid are rare, the keratin origin of amyloid has been demonstrated in lichen amyloidosis and macular amyloidosis [18]. The damage may then result in keratinocyte deposition and degenerate to amyloid at the dermo-epidermal junction [18]. Only 3 reports in the literature reported the keratin composition of the amyloid in ACD $[5,12,16]$. However, only wide-spectrum cytokeratin antibodies or high molecular weight cytokeratin antibodies were used in these reports. Wide-spectrum anti-cytokeratin antibodies were 


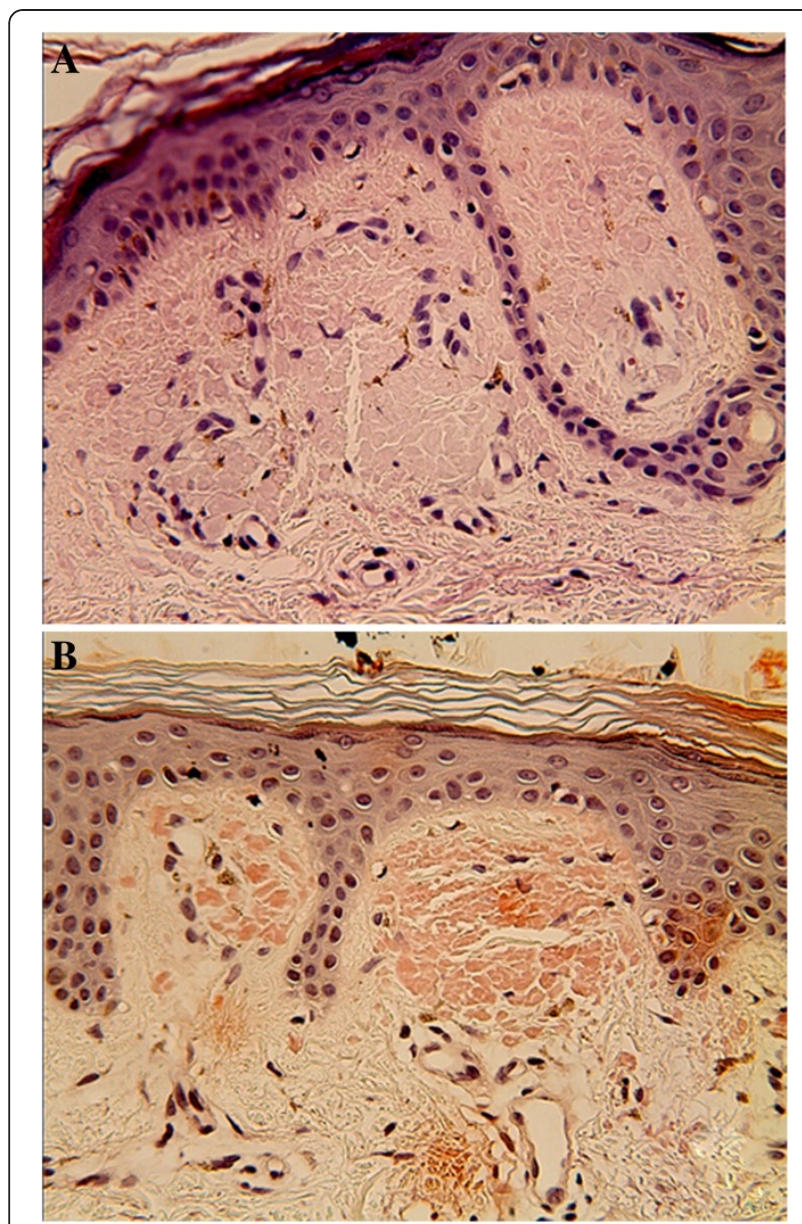

Figure 3 Histopathological manifestation of amyloidosis cutis dyschromica. A, Histopathology of amyloidosis cutis dyschromica skin lesions showed homogenous eosinophilic masses in the papillary dermis and sparse melanophages in the superficial dermis (patient 1b; hematoxylin-eosin, original magnification: $\times 400$ ). B, The Congo red stain showed positivity in the papillary dermal deposits (original magnification $\times 400$ magnification).

positive in two of the three cases [12,16], and high molecular weight cytokeratin was positive in another patient [5]. In our series, $100 \%(6 / 6)$ of the cases showed immunohistochemical positivity for CK34ßE12 and CK5/6 and negative or faint positive staining for pan-cytokeratin AE1/AE3. To our knowledge, this is the first report showing the amyloid staining profile of CK34ßE12 and CK5/6 in ACD. The immunohistopathological results and limited literature reports strongly support an epidermal origin of the amyloid.

The occurrence of familial cases and disease onset before puberty suggests a genetic aetiology of ACD. The genetic basis of ACD is not known. It has been suggested that genetic factors may lead to the disruption of DNA repair in keratinocytes [16]. Moriwaki et al. observed slow DNA repair in keratinocytes due to ultraviolet $\mathrm{C}$ damage and reduced repair following ultraviolet

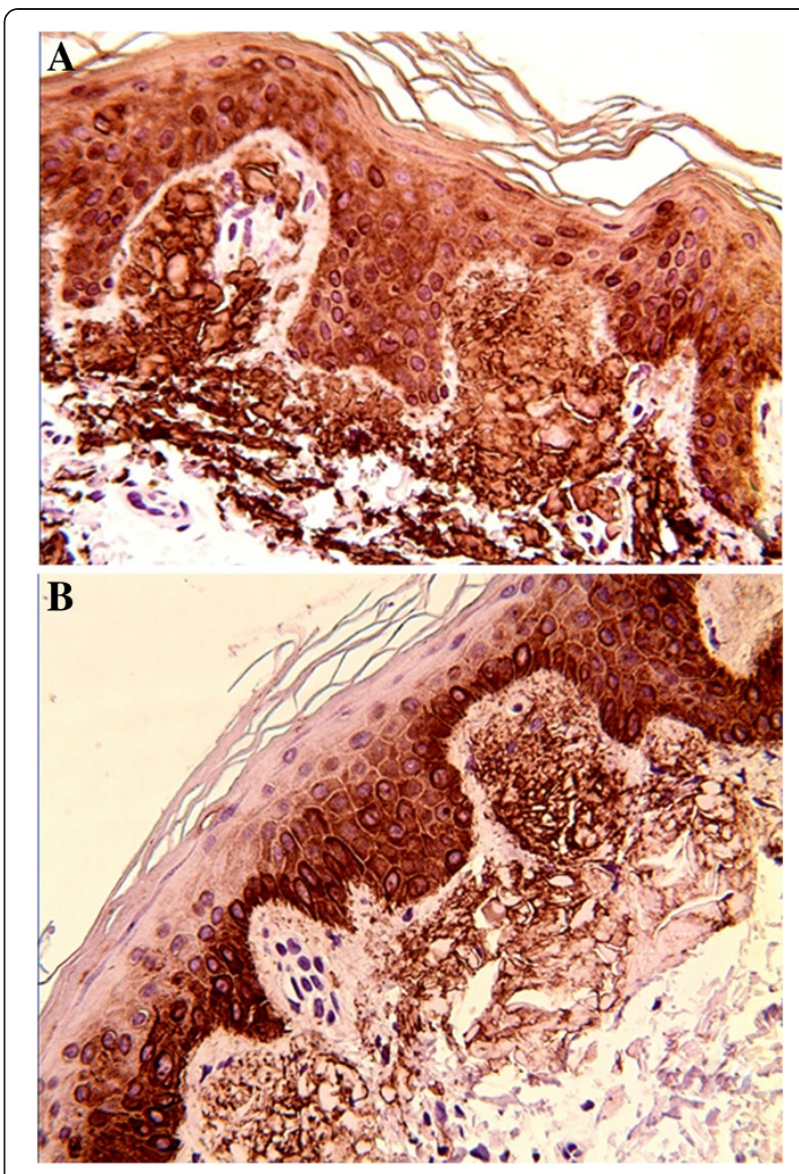

Figure 4 Immunohistochemical profile of amyloidosis cutis dyschromica. Cytokeratin staining showed that the papillary dermal deposits were strongly positive for CK34ßE12 and CK5/6 in amyloidosis cutis dyschromica. (patient 5; A, CK34ßE12 stain; B, CK5/ 6 stain; original magnifications: $\mathbf{A}, \times 400, \mathbf{B}, \times 400$ ).

B damage [16]. However, the cutaneous findings of all patients are distributed over the entire skin surface, and the face, neck, and hands often have a milder presentation than the non-sun-exposed skin area. This argues against a primary role of photosensitivity in this disorder.

The differential diagnosis of dyschromatoses is often a challenge to clinical dermatologists, particularly in ethnic populations in which this disease is not commonly observed [19]. The differential diagnosis of ACD includes other diseases presenting as dyschromatosis and other amyloid deposition disorders, including dyschromatosis universalis hereditaria, xeroderma pigmentosum, and poikiloderma-like cutaneous amyloidosis [20]. Dyschromatosis universalis hereditaria is also characterised by the presence of generalised hypopigmented and hyperpigmented macules, and it begins before puberty. However, histologically, no amyloid deposition has been observed in dyschromatosis universalis hereditaria [21]. Xeroderma pigmentosum may have a similar dyschromatosis appearance but with 


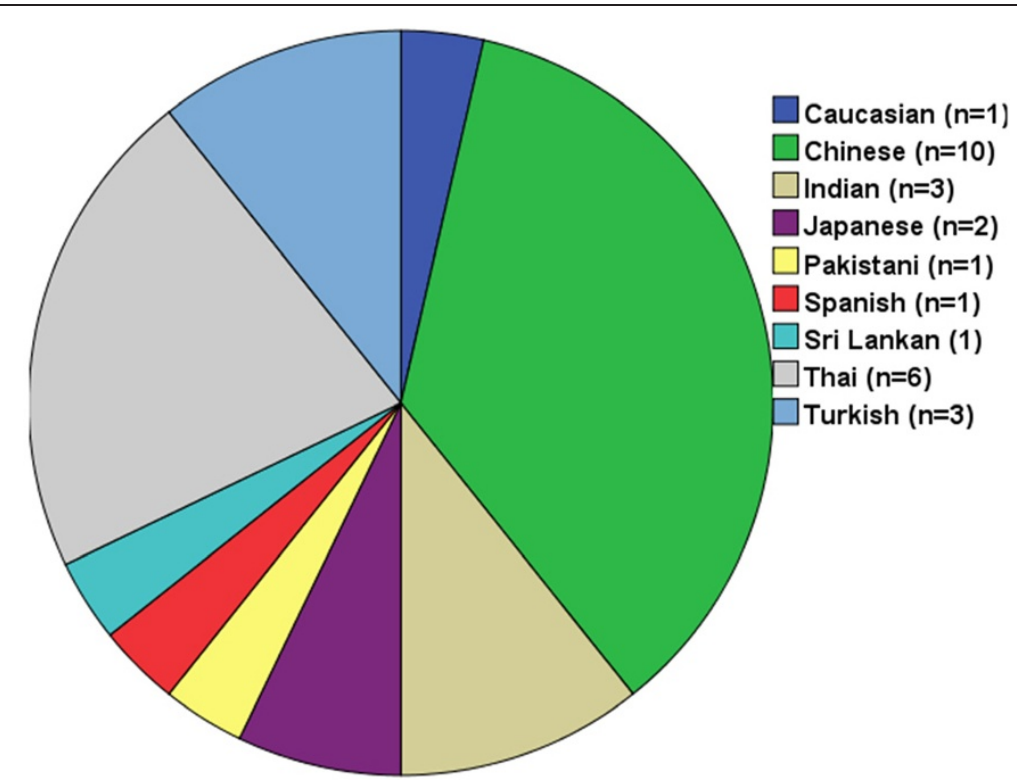

Figure $\mathbf{5}$ Ethnic distribution of $\mathbf{2 6}$ cases of amyloidosis cutis dyschromica from the literature. Most published cases of amyloidosis cutis dyschromica observed in Asian patients.

marked photosensitivity [22]. There is also evidence of actinic damage beginning in infancy and early childhood, and skin cancers often develop during the first decade of life [22]. The clinical features of poikiloderma-like cutaneous amyloidosis are similar to those of ACD [23]. Poikiloderma-like cutaneous amyloidosis is characterised by the presence of reticular hyper- and hypopigmentation lesions, atrophy and telangiectasia. However, many of the published patients did not fully express the triad. Diffuse hyperpigmentation was reported in all described patients, with telangiectasia, atrophy and lichenoid papules in some. This variant is associated with photosensitivity, short stature and palmoplantar keratoderma [23,24]. A few clinical features overlap between poikiloderma-like cutaneous amyloidosis and ACD. For example, dotty atrophy and vesicles were observed in two siblings with ACD [3], and telangiectasia and photosensitivity were observed in one patient with ACD [16].

The treatment of ACD is not well documented. Success has been reported with photoprotection, antioxidants and acitretin in some case reports [12]. We used acitretin in three patients in our series, and a significant improvement of the skin appearance was observed in two. Therefore, acitretin may be a promising drug for treating the disease.

A major limitation of our series and the published literature regarding ACD continues to be small sample size. $\mathrm{ACD}$ is a rare disease; therefore, it is very difficult to identify sample sizes large enough to reach significant conclusions.

\section{Conclusions}

We report 10 new cases of ACD. The distinguishing features of the clinical presentation included generalised mottled hyper- and hypopigmented macules. No evidence of systemic amyloidosis deposition was found in these patients. Amyloid deposits were observed in the papillary dermis. Positive staining for cytokeratins CK34ßBE12 and CK5/6 in amyloidosis cutis dyschromica suggests that the amyloid is derived from keratinocytes.

\section{Additional files}

Additional file 1: Table S1. The manufacturers of the antibodies used in this study and the keratins recognised.

Additional file 2: Table S2. Clinical and histological data of patients with amyloidosis cutis dyschromica: current series.

Additional file 3: Table S3. Clinical and histological data of patients with amyloidosis cutis dyschromica: a review of published cases.

Abbreviations

ACD: Amyloidosis cutis dyschromica; CK: Cytokeratin.

\section{Competing interests}

All authors declare that they have no competing interests.

\section{Authors' contributions}

$J Q$ and HF collected the clinical data. JQ drafted the manuscript. HY carried out the pathological and immunohistochemical assays. JQ, HF, and $\mathrm{HY}$ participated in the design of the study. JQ conceived of the study. HF and HY revised the manuscript. All authors read and approved the final manuscript. 


\section{Acknowledgment}

This study was supported by the National Natural Science Foundation of China (30900056).

\section{Author details}

'Department of Dermatology, The First Affiliated Hospital, College of Medicine, Zhejiang University, No. 79, Qingchun Road, Hangzhou 310003, Zhejiang Province, China. ${ }^{2}$ Department of Pathology, The First Affiliated Hospital, College of Medicine, Zhejiang University, No. 79, Qingchun Road, Hangzhou 310003, Zhejiang Province, China.

Received: 9 August 2012 Accepted: 30 November 2012

Published: 12 December 2012

\section{References}

1. Schreml S, Szeimies RM, Vogt T, Landthaler M, Schroeder J, Babilas P: Cutaneous amyloidoses and systemic amyloidoses with cutaneous involvement. Eur J Dermatol 2010, 20:152-160.

2. Morishima T: A clinical variety of localized cutaneous amyloidosis characterized by dyschromia (amyloidosis cutis dyschromica). Jpn J Dermatol Series B 1970, 80:43-52.

3. Yang W, Lin Y, Yang J, Lin W: Amyloidosis cutis dyschromica in two female siblings: cases report. BMC Dermatol 2011, 11:4.

4. Garg T, Chander R, Jabeen M, Barara M, Mittal K, Jain M, Puri V: Amyloidosis cutis dyschromica: a rare pigmentary disorder. J Cutan Pathol 2011, 38:823-826.

5. Fernandes NF, Mercer SE, Kleinerman R, Lebwohl MG, Phelps RG: Amyloidosis cutis dyschromica associated with atypical Parkinsonism, spasticity and motor weakness in a Pakistani female. J Cutan Pathol 2011, 38:827-831.

6. Chandran NS, Goh BK, Lee SS, Goh CL: Case of primary localized cutaneous amyloidosis with protean clinical manifestations: lichen, poikiloderma-like, dyschromic and bullous variants. J Dermatol 2011, 38:1066-1071.

7. Madarasingha NP, Satgurunathan K, De Silva MV: A rare type of primary cutaneous amyloidosis: amyloidosis cutis dyschromica. Int I Dermatol 2010, 49:1416-1418.

8. Karadağ AS, Şimşek GG: Familial amyloidosis cutis dyschromica. Turk J Med Sci 2010, 40:151-154

9. Huang WH, Wu CY, Yu CP, Chiang CP: Amyloidosis cutis dyschromica: four cases from two families. Int J Dermatol 2009, 48:518-521.

10. Ho MS, Ho J, Tan SH: Hypopigmented macular amyloidosis with or without hyperpigmentation. Clin Exp Dermatol 2009, 34:e547-e551.

11. Wu CY, Yu CP, Chiang CP: Familial amyloidosis cutis dyschromica-a case report and review of the literature. Dermatol Sinica 2008, 26:16-21.

12. Ozcan A, Senol M, Aydin NE, Karaca S: Amyloidosis cutis dyschromica: a case treated with acitretin. J Dermatol 2005, 32:474-477.

13. Morales Callaghan AM, Vila JB, Fraile HA, Romero AM, Garcia GM: Amyloidosis cutis dyschromica in a patient with generalized morphoea. Br J Dermatol 2004, 150:616-617.

14. Choonhakarn C, Wittayachanyapong S: Familial amyloidosis cutis dyschromica: six cases from three families. J Dermatol 2002, 29:439-442.

15. Vijaikumar M, Thappa DM: Amyloidosis cutis dyschromica in two siblings. Clin Exp Dermatol 2001, 26:674-676.

16. Moriwaki S, Nishigori C, Horiguchi Y, Imamura S, Toda K, Takebe H: Amyloidosis cutis dyschromica. DNA repair reduction in the cellular response to UV light. Arch Dermatol 1992, 128:966-970.

17. Eng AM, Cogan L, Gunnar RM, Blekys I: Familial generalized dyschromic amyloidosis cutis. J Cutan Pathol 1976, 3:102-108.

18. Fernandez-Flores A: Cutaneous amyloidosis: a concept review. Am J Dermatopathol 2012, 34:1-14. quiz 15-17.

19. Urabe K, Hori Y: Dyschromatosis. Semin Cutan Med Surg 1997, 16:81-85.

20. Vachiramon V, Thadanipon K, Chanprapaph K: Infancy- and childhood-onset dyschromatoses. Clin Exp Dermatol 2011, 36:833-838. quiz 839.

21. Sethuraman G, Thappa DM, Vijaikumar, Kumar J, Srinivasan S: Dyschromatosis universalis hereditaria: a unique disorder. Pediatr Dermatol 2000, 17:70-72.

22. Kraemer KH, Lee MM, Scotto J: Xeroderma pigmentosum. Cutaneous, ocular, and neurologic abnormalities in 830 published cases. Arch Dermatol 1987, 123:241-250.
23. Pardo Arranz L, Escalonilla Garcia-Patos P, Roman Curto C, Blanco Barrios S, Fernandez Lopez E, de Unamuno Perez P: Familial poikylodermic cutaneous amyloidosis. Eur J Dermatol 2008, 18:289-291.

24. Ogino A, Tanaka S: Poikiloderma-like cutaneous amyloidosis. Report of a case and review of the literature. Dermatologica 1977, 155:301-309.

doi:10.1186/1750-1172-7-95

Cite this article as: Qiao et al: Amyloidosis cutis dyschromica. Orphanet Journal of Rare Diseases 2012 7:95.

\section{Submit your next manuscript to BioMed Central and take full advantage of:}

- Convenient online submission

- Thorough peer review

- No space constraints or color figure charges

- Immediate publication on acceptance

- Inclusion in PubMed, CAS, Scopus and Google Scholar

- Research which is freely available for redistribution

Submit your manuscript at www.biomedcentral.com/submit
C Biomed Central 